der Bevölkerung“ neben die „Erhaltung der natürlichen Lebensgrundlagen und die Pflege der Kulturlandschaft". Im Grundgesetz stehen die beiden auch von der Erwiderung hervorgehobenen Aspekte der Ernährungssicherheit und des Schutzes der natürlichen Lebensgrundlagen mit Art. 20a und Art. 74 Abs. 1 Nr. 17 GG weit auseinander und lassen zudem als Staatszielbestimmung einerseits und Kompetenzzuweisungsnorm andererseits anders als in der schweizerischen Bundesverfassung keine direkte Beziehung zueinander erkennen. Die von der Erwiderung hervorgehobene und als regelungsbedürftig angesehene Funktion des Landwirts als Landschaftspfleger ${ }^{66}$ taucht anders als in der schweizerischen Bundesverfassung im Grundgesetz in Verbindung mit der Landwirtschaft überhaupt nicht auf.

Wird zum Schluss noch einmal zum BLwG zurückgekehrt, so sollte ein solcher Gesetzestitel nach Ansicht des Verfassers einem Gesetz vorbehalten bleiben, das die Landwirtschaft nicht nur unter dem Aspekt eines Sachbereichs, sondern breiter angelegt erfasst. Insofern können das SchweizLwG und der Code rural, aber zum Beispiel auch das Bayerische Agrarwirtschaftsgesetz als Anschauungsmaterial dienen. Wie weitgehend in einem solchen Gesetz das bestehende Agrarrecht zusammengefasst sowie integrierter ausgestaltet wird und in welchem Umfang der Bereich des ländlichen Raumes mit zu erfassen ist, wäre näher zu ergründen. Hierfür kann auf den Optionen, die in dem Bericht von 2008 zur Revision des BLwG dargestellt wurden, aufgebaut werden.

Die Überführung von Bestimmungen, die in einem festen systematischen Kontext anderer Gesetzeswerke stehen, erscheint allerdings prinzipiell problematisch, wie vorliegend anhand des Agrarumweltrechts erläutert wurde. In solchen Fällen empfiehlt sich eher die Nutzung einer Verweisungstechnik. Auch die Aufnahme von Gesetzen, die nicht nur auf die Landwirtschaft anwendbar sind, bleibt schwierig. $\mathrm{Zu}$ nennen sind etwa das Pflanzenschutzgesetz und das Düngegesetz, da sie an erster Stelle die Herstellung und Vermarktung der betreffenden Stoffe regeln und insofern zum Rechtsgebiet des Gefahrstoffrechts gehören. Außerdem ist die Regulierung der Anwendung der Stoffe nicht lediglich auf die Landwirtschaft beschränkt, sondern umfasst auch den privaten Gebrauch.

$\mathrm{Ob}$ im Rahmen eines revidierten BLwG allgemeingültige Definitionen der agrarrechtlichen Grundbegriffe - Land- wirtschaft, Landwirt, landwirtschaftlicher Betrieb und landwirtschaftliche Fläche - geschaffen werden sollten, ist eine reizvolle Frage und müsste eingehender in Bezug auf die verschiedenen Anwendungsbereiche der Grundbegriffe geprüft werden. Ähnlich gelagert ist die Frage der Einführung eines Konzeptes von Betreiberpflichten für landwirtschaftliche Betriebe. Hier bedürfte es zunächst der Klärung, ob damit lediglich eine Zusammenfassung bestehender Pflichten oder aber ein neues Ordnungskonzept gewollt ist, das in die Richtung geht, jeden landwirtschaftlichen Betrieb als eine Anlage zu betrachten, die einer Grundgenehmigung und beständigen Überprüfung, ob die Voraussetzungen der Grundgenehmigung noch gegeben sind, bedarf. Eine knifflige Frage ist zudem die Verzahnung mit dem agrarrechtlichen Landesrecht, das unter anderem das mit dem landwirtschaftlichen Anerbenrecht, dem Grundstückverkehrsrecht, dem Flurbereinigungsrecht, dem Siedlungsrecht und dem nach Art. 72 Abs. 3 GG möglichen Abweichungsrecht wichtige Felder des Agrarrechts berührt. Alles in allem bietet sich hinreichender Stoff für eine weitere spannende Diskussion der Thematik.

Open Access. Dieser Artikel wird unter der Creative Commons Namensnennung 4.0 International Lizenz veröffentlicht, welche die Nutzung, Vervielfältigung, Bearbeitung, Verbreitung und Wiedergabe in jeglichem Medium und Format erlaubt, sofern Sie den/die ursprünglichen Autor(en) und die Quelle ordnungsgemäß nennen, einen Link zur Creative Commons Lizenz beifügen und angeben, ob Änderungen vorgenommen wurden.

Die in diesem Artikel enthaltenen Bilder und sonstiges Drittmaterial unterliegen ebenfalls der genannten Creative Commons Lizenz, sofern sich aus der Abbildungslegende nichts anderes ergibt. Sofern das betreffende Material nicht unter der genannten Creative Commons Lizenz steht und die betreffende Handlung nicht nach gesetzlichen Vorschriften erlaubt ist, ist für die oben aufgeführten Weiterverwendungen des Materials die Einwilligung des jeweiligen Rechteinhabers einzuholen.

Weitere Details zur Lizenz entnehmen Sie bitte der Lizenzinformation auf http://creativecommons.org/licenses/by/4.0/deed.de.

Open Access funding enabled and organized by Projekt DEAL.

66) Czybulka/Fischer-Hüftle/Hampicke/Köck/Martinez (Fn. 3), S. 303.

\title{
Kastenstandhaltung und kein Ende
}

\section{Claudia Schürmeier}

(C) Der/die Autor(en) 2021. Dieser Artikel ist eine Open-Access-Publikation.

Seit Jahrzehnten werden Jungsauen und Sauen über Wochen hinweg in Kastenständen zur Zucht von Ferkeln eingepfercht und können sich weder bewegen noch umdrehen. Selbst das Ausstrecken der Beine in Seitenlage wird zum jahrelangen Diskussionsgegenstand, weil aus wirtschaftlichen Gründen um die Breite der Kastenstände und damit um die baulichen Bedingungen in den Ställen gerungen wird. ${ }^{1}$ Die Stimmen in der Gesellschaft, die sich gegen eine solche Behandlung der Schweine, aber auch gegen den in gro-

Dr. jur. Claudia Schürmeier, Dipl.-Verw. (FH),

Lehrbeauftragte Fachbereich Verwaltungswissenschaften,

Hochschule Harz,

Halberstadt, Deutschland ßen Teilen nicht artgerechten Umgang mit Tieren in der Landwirtschaft insgesamt wenden, wurden in den vergangenen Jahren immer lauter. Werden politische Entscheidungen zugunsten der Tiere getroffen, sind diese bis zu ihrer Wirksamkeit allerdings oftmals mit langen Übergangsfristen verbunden. Ein Beispiel dafür ist die Beschlussfassung der Siebten Änderungsverordnung zur TierschutzNutztierhaltungsverordnung, die die rechtliche Grundlage für die

1) Schweine sind nicht die einzige Tierart, die zumindest zeitweise in Käfigen gehalten werden. Hier sei beispielhaft nur auf die Käfighaltung von Kaninchen, insbesondere in der konventionellen Kaninchenmast hingewiesen; Hirt/Maisack/Moritz, TierSchG, 3. Aufl. 2016, TierSchNutztV, vor \$31-37, Rdnr. 12 ff. 
Abschaffung der Kastenstandhaltung legt. Dennoch ist der jüngste Beschluss des Bundesrates aus tierschutzrechtlicher Sicht keineswegs zufriedenstellend. Die Änderungsverordnung vermittelt den Eindruck, es gehe in erster Linie nicht um das Wohl der Tiere, sondern um ein möglichst langes Hinauszögern der Abschaffung der Kastenstandhaltung, um wirtschaftliche Nachteile für die Halter gering zu halten. Übergangsfristen von bis zu fünfzehn Jahren nach Inkrafttreten der Verordnung geben den Schweinehaltern eine lange Planungsperspektive. Für die betroffenen Tiere ändert sich derweil auf Jahre hinaus aber nichts.

\section{Zum Sachstand}

Tierschützer ringen seit Jahrzehnten um die Abschaffung der Kastenstandhaltung von Sauen, eine Haltungsform, die nachweislich mit massiven Einschränkungen der Tiere in ihrem natürlichen Verhalten verbunden ist und zu körperlichen und psychischen Schäden führt. In Deutschland wurden im Jahr 2019 ca. 1,8 Mio. Zuchtsauen ${ }^{2}$ gehalten, die einen Großteil ihres Lebens in einem Kastenstand verbrachten.

Die Tierschutz-Nutztierhaltungsverordnung (TierSchNutztV) ${ }^{3}$ schreibt vor, wie solche Kastenstände beschaffen sein müssen. Jedoch ist fraglich, ob diese Regelung nicht bereits tierschutzrechtswidrig ist, weil sie eine nicht artgerechte Haltung von Schweinen legitimiert. Aber selbst die in $\$ 24$ Abs. 4 TierSchNutztV a. F. formulierten Erfordernisse an die Beschaffenheit von Kastenständen werden seit Jahrzehnten durch die zahlreichen Schweinehaltungsbetriebe missachtet. ${ }^{4}$ Spätestens seit der Entscheidung des Bundesverwaltungsgerichts ${ }^{5}$ aus dem Jahre 2016 sind die verordnungsrechtlich geregelten Anforderungen an die Beschaffenheit von Kastenständen $(\$ 24$ Abs. 4 Nr. 2 TierSchNutztV a. F.) höchstrichterlich als rechtsverbindlich bestätigt worden.

Die Politik reagierte zwar auf die rechtswidrigen $\mathrm{Zu}-$ stände in den Schweineställen wiederholt mit gesetzgeberischen Aktivitäten. Diese zeichnen sich allerdings allenfalls durch lange Übergangsfristen aus.

Die jüngste Ânderungsverordnung verbessert kaum etwas an der gegenwärtigen Situation der Tiere und legitimiert, dass die Schweine noch auf Jahre hinaus in Kastenständen eingesperrt werden. Vielmehr muss eine Abschaffung der Kastenstandhaltung, wie sie auch schon in anderen europäischen Ländern vorgenommen wurde, ${ }^{6}$ auch in Deutschland schnellstmöglich erfolgen, um das Leid der Tiere zu beenden. Es kann also nicht um das „Wie“ der Kastenstandhaltung gehen, sondern nur um das „Ö“.

Das Thema der Kastenstandhaltung wurde in den vergangenen Jahren in der juristischen Fachliteratur wiederholt thematisiert. ${ }^{7}$ Auf dieser Grundlage reflektiert der vorliegende Beitrag den aktuellen rechtlichen Stand in diesem Bereich der Schweinehaltung und betrachtet diesen im Lichte der aktuellen Siebten Verordnung zur Änderung der Tierschutz-Nutztierhaltungsverordnung.

Zunächst gibt der Beitrag einen kurzen Einblick in die unterschiedlichen Ausprägungen der Kastenstandhaltung und geht auf die gesundheitlichen, physischen wie psychischen Folgen für die Tiere ein (Abschnitt 2). In dem folgenden Abschnitt wird die Rechtmäßigkeit der Kastenstandhaltung betrachtet. Dabei geht es sowohl um die verfassungsrechtlichen Rahmenbedingungen, um einfachgesetzliche tierschutzrechtliche Regelungen als auch um die Rechtsprechung zur Beschaffenheit von Kastenständen. Sodann gilt das Augenmerk der jüngst in Kraft getretenen Siebten Änderungsverordnung. Betrachtet werden dabei die Änderungen der Regelungen zur Kastenstandhaltung und die Übergangsfristen bis zu ihrer weitgehenden Abschaffung (Abschnitt 4). Nicht zuletzt nimmt der Beitrag einen Normenkontrollantrag des Landes Berlin zur Überprüfung der Anforderungen an die Schweinehaltung in den Blick (Abschnitt 5). Der Beitrag schließt mit einer Zusammenfassung und einem Ausblick (Abschnitt 6).

\section{Haltungsformen im Kastenstand und Tierleid}

Seit Jahrzehnten werden Sauen mit unterschiedlicher Zwecksetzung über einen Zeitraum von Monaten hinweg in sog. Kastenständen gehalten. Bislang legalisierte $\ 30$ Abs. 2 TierSchNutztV a. F. die Kastenstandhaltung, indem er festlegte, dass Sauen lediglich in einem Zeitraum von über vier Wochen nach dem Decken bis eine Woche vor dem voraussichtlichen Abferkeltermin in Gruppen gehalten werden müssen. ${ }^{8}$ Aufgrund einer entsprechenden Übergangsregelung wird dies für die nächsten Jahre auch so weitergelten. ${ }^{9}$ Im Umkehrschluss werden die Sauen eine Woche vor dem Abferkeltermin bis zum Absetzen der Ferkel im Kastenstand fixiert und verbleiben dort bis zum Ablauf von vier Wochen nach dem Decken. ${ }^{10}$ Die Haltung der Tiere in den Kastenständen wird also vom Verordnungsgeber bei bestimmter Beschaffenheit des Kastenstandes akzeptiert.

Bei der Kastenstandhaltung geht es demnach zum einen um den Deckvorgang zum anderen um das sog. Abferkeln. Eine Differenzierung in der Betrachtung ist insofern sinnvoll, als beide Phasen der Kastenstandhaltung an unterschiedliche rechtliche Rahmenbedingungen geknüpft sind.

\subsection{Haltungsbedingungen im Deckzentrum}

Im Deckzentrum wird die Sau vom Zeitpunkt des Absetzens bzw. Deckens bis zum 28. Tag nach dem Decken in einem sog, Besamungsfressstand permanent fixiert, einem bis $\mathrm{zu} 2 \mathrm{~m}$ langen und $0,7 \mathrm{~m}$ breiten Kastenstand, der mit einem teilperforierten Boden ausgestattet ist. ${ }^{11}$ Durch die permanente Fixierung sind nahezu alle Grundbedürfnisse der Tiere, wie beispielsweise das Schlafen oder die Körperpflege, stark eingeschränkt, so dass ein erhöhtes Risiko für Verhaltensstörungen, wie Stangenbeißen und Leerkauen, sowie Erkrankungen des Herz-Kreislauf-Apparates besteht. Eine Fortbewegung ist gänzlich ausgeschlossen. ${ }^{12}$

Begründet wird die Kastenstandhaltung im Deckzentrum mit der Übersichtlichkeit für das Personal und der Verhinderung des Abgangs der Frucht. ${ }^{13}$

2) ISN-Interessengemeinschaft der Schweinehalter Deutschlands e.V., Schweinebestände in Deutschland seit 2010, Stand 20.6.2020, abrufbar unter https://www.schweine.net/markt/ strukturdaten.html.

3) Verordnung zum Schutz landwirtschaftlicher Nutztiere und anderer zur Erzeugung tierischer Produkte gehaltener Tiere bei ihrer Haltung, i.d.F. der Bekanntmachung v. 22.8.2006, BGBl. I S. 2043; zuletzt geändert durch Art. 1a der Verordnung v. 29.1.2021, BGBl. I S. $146 f$.

4) Vgl. BR-Drs. 587/19, S. 1 f. Felde, Rechtsgutachten zum Referentenentwurf des Bundesministeriums für Ernährung und Landwirtschaft zu einer Siebten Verordnung zur Änderung der Tierschutz-Nutztierhaltungsverordnung, Entwurfsstand: 28.5.2019, abrufbar unter https://www.djgt.de/news/20200628172810_ 20190702_Felde_StN_zu_Referentenentwurf.pdf, S. 53.

5) BVerwG, Beschl. v. 8.11.2016, Az. 3B 11.16.

6) In den skandinavischen Ländern ist die Kastenstandhaltung seit 1988 verboten. So: Ziron, Schweinezucht und Schweinemast (SUS) 3/2018, S. 18, 19.

7) Vgl. Wollenteit/Lemke, NuR 2013, 177 ff.; Maisack, NuR 2017, $456 \mathrm{ff}$.

8) Zuchtsauen haben eine Trächtigkeit von 115 Tagen. Sie werden durchschnittlich 2,5-mal im Jahr befruchtet und verbringen daher insgesamt ca. 6 Monate im Jahr in Kastenständen. Wollenteit/ Lemke, NuR 2013, 177, 178.

9) Vgl. unten Abschnitt 4.3.

10) Hirt/Maisack/Moritz, TierSchG, TierSchNutztV, vor $₫ 30$, Rdnr. 1 .

11) Hirt/Maisack/Moritz, TierSchG, TierSchNutztV, vor $\$ \$ 21-30$, Rdnr. 20.

12) Hirt/Maisack/Moritz, TierSchG, TierSchNutztV, vor $\$ \$ \$ 21-30$, Rdnr. 20 m.w.N.; Kuratorium für Technik und Bauwesen in der Landwirtschaft (Hrsg.), Nationaler Bewertungsrahmen Tierhaltungsverfahren, Intensivdeckzentrum S/FD0001, S. 3 f.

13) Felde, Rechtsgutachten, S. 4. 
Ein tierschutzrechtliches Kernproblem der Kastenstandhaltung im Deckzentrum ist das Ausstrecken der Gliedmaßen. $\$ 24$ Abs. 4 Nr. 2 TierSchNutztVO a.F. regelte bisher, dass der Kastenstand so beschaffen sein muss, dass jedes Schwein in Seitenlage die Gliedmaßen ausstrecken kann. In der Praxis sind die Kastenstände aber oftmals so schmal, dass ein Ausstrecken der Gliedmaßen nicht möglich ist. Sofern der benachbarte Kastenstand ebenfalls belegt ist, stößt die Sau mit ihren Gliedmaßen an den Rücken der benachbarten Sau.

\subsection{Haltungsbedingungen in einer Abferkelbucht}

Sieben Tage vor der Geburt der Ferkel wird die Sau erneut in einen Kastenstand in einer Abferkelbucht verbracht, wo sie ihre Ferkel zur Welt bringt und dort vom 21. bis 30. Tag nach dem Abferkeln verbleibt, also nach dem Monat im Deckzentrum weitere fünf Wochen in der Kastenstandhaltung. ${ }^{14}$ Wie bei der Kastenstandhaltung im Deckzentrum ist in diesem Kastenstand auch kein Umdrehen möglich. Durch die zwangsweise Immobilisierung der Tiere kommt es zu einer signifikanten Verlängerung des Geburtsvorganges. ${ }^{15}$ Auch das zur Geburtsvorbereitung arttypische starke Bedürfnis nach Nestbauverhalten wird weitgehend unterdrückt. ${ }^{16}$ Anders als bei der Kastenstandhaltung im Deckzentrum ist aber zumindest ein Ausstrecken der Gliedmaßen möglich, da sich der Kastenstand in der Mitte der Abferkelbucht befindet und die Sau die Gliedmaßen in den Bereich ausstrecken kann, in dem sich die Ferkel aufhalten. ${ }^{17}$ Dennoch ist das Mutter-Kind-Verhalten stark eingeschränkt. ${ }^{18}$

Die Fixierung von Sauen wird in erster Linie damit begründet, dass in den ersten Tagen nach der Geburt ohne eine Fixierung der Sau ein hohes Erdrückungsrisiko für die Ferkel bestehe. Zudem biete die Fixierung aber auch arbeitsökonomische Vorteile sowie Vorteile in Bezug auf den Arbeitsschutz. ${ }^{19}$

\section{Zur Rechtmäßigkeit der Kastenstandhaltung}

Seit Jahrzehnten gehört die Kastenstandhaltung zum Alltag in der Schweinezucht. Mit einem zunehmenden gesellschaftlichen Wandel zugunsten des Tierschutzes ist auch die Kritik an der Haltungsform des Kastenstandes in den vergangenen Jahren gewachsen. Daher stellt sich einmal mehr die Frage nach der Vereinbarkeit der Kastenstandhaltung mit dem in Art. 20a GG verankerten Staatsziel Tierschutz und den einfachgesetzlichen Regelungen zur Kastenstandhaltung.

\subsection{Verfassungsrechtliche Rahmenbedingungen}

Gem. Art. 20a GG sind die Tiere im Rahmen der verfassungsmäßigen Ordnung durch die Gesetzgebung und nach Maßgabe von Gesetz und Recht durch die vollziehende Gewalt und die Rechtsprechung geschützt. ${ }^{20}$

Eines der Gewährleistungselemente des an Bund und Länder gerichteten Staatsziels besteht im Schutz der Tiere vor nicht artgemäßer Haltung. ${ }^{21}$ Es sollen also Haltungsformen gewährleistet werden, die den Anforderungen gem. $\$ 2$ TierSchG entsprechen, mithin eine art- und bedürfnisangemessene Ernährung, Pflege und verhaltensgerechte Unterbringung. Demnach soll es zumindest mittelfristig keine Haltungssysteme mehr geben, in denen die Grundbedürfnisse der Tiere weiterhin unangemessen zurückgedrängt werden. ${ }^{22}$

Das Staatsziel Tierschutz enthält eine Werterhöhung, die eine gestiegene Verantwortung für das Tier impliziert. ${ }^{23}$ Dabei geht eine Werterhöhung u.a. einher mit einer stärkeren Rechtfertigungsbedürftigkeit von Handlungen, die Tiere belasten, einem größeren Gewicht des Tierschutzes bei Abwägungsvorgängen und der Pflicht des Staates zu Verbesserungen beim Tierschutz und zur Verhinderung von Verschlechterungen. ${ }^{24}$

Das vom Staatsziel Tierschutz umfasste Verbesserungsgebot impliziert eine permanente staatliche Nachbesserungs- pflicht. So ist der Gesetz- und Verordnungsgeber gehalten, bestehende Tierschutznormen an neue wissenschaftliche Erkenntnisse zur Empfindungs- und Leidensfähigkeit der Tiere und an einen Wandel der gesellschaftlichen Sensibilität und der ethischen Standards anzupassen. ${ }^{25}$ Dieser Wandel ist in den vergangenen Jahren deutlich feststellbar. Die Einstellung zu Tieren, insbesondere auch im Bereich der industriellen Massentierhaltung, hat sich maßgeblich geändert. Zugleich werden die Zustände in den Agrarbetrieben und der Umgang der Tierhalter mit den Nutztieren zunehmend hinterfragt und missbilligt. ${ }^{26}$

Die Staatszielbestimmung wirkt zugleich wie ein grundsätzliches Verschlechterungsverbot. ${ }^{27}$ So wird der Gesetzund Verordnungsgeber verpflichtet, bei Novellierungen des Tierschutzgesetzes nicht hinter geltendes Recht zurückzugehen. ${ }^{28}$

Das Staatsziel Tierschutz ist mit anderen Verfassungsgütern grundsätzlich gleichrangig. Daher kann weder zugunsten der Grundrechte noch zugunsten des Tierschutzes von einem generellen Vorrang ausgegangen werden. Vielmehr ist im Wege der Abwägung zu entscheiden, welches Gut zurückzutreten hat. ${ }^{29}$ Konkurrenzlagen zwischen dem Staatsziel Tierschutz und betroffenen Grundrechten wie Art. 12 GG und Art. 14 GG sind nach dem Prinzip der praktischen Konkordanz miteinander in Ausgleich zu bringen. ${ }^{30}$

\subsection{Der einfachgesetzliche Rechtsrahmen}

Die Kastenstandhaltung unterliegt als eine landwirtschaftliche Haltungsform von Tieren den Tierhaltungsbedingungen des $\$ 2$ TierSchG. Danach muss der Halter von Tieren, insbesondere das Tier seiner Art und seinen Bedürfnissen entsprechend angemessen ernähren, pflegen und verhaltensgerecht unterbringen ( $\$ 2$ Nr. 1 TierSchG). Er darf die artgemäße Bewegung nicht so einschränken, dass dem Tier Schmerzen oder vermeidbare Leiden oder Schäden zugefügt werden.

Ermächtigungsgrundlage der Tierschutz-Nutztierhaltungsverordnung ist $\$ 2$ a TierSchG, die es dem Bundesministerium für Ernährung und Landwirtschaft erlaubt, durch Rechtsverordnung die Anforderung an die Haltung von Tieren nach $₫ 2$ TierSchG näher zu bestimmen.

Die Kastenstandhaltung ist unvereinbar mit $\$ 2$ TierSchG. ${ }^{31}$ Sie verstößt insbesondere gegen die in $\$ 2 \mathrm{Nr} .1$

14) Felde, Rechtsgutachten, S. 4

15) Maisack, NuR 2017, 456, 460 m.w. N.

16) Maisack, $\mathrm{NuR} 2017,456,460$.

17) Felde, Rechtsgutachten, S. 4

18) Hirt/Maisack/Moritz, TierSchG, TierSchNutztV, vor $\$ \$ 21-30$, Rdnr. 18.

19) Deutscher Bundestag, Antwort der Bundesregierung auf die Kleine Anfrage der Abgeordneten Friedrich Ostendorff, Harald Ebner, Renate Künast, weiterer Abgeordneter und der Fraktion BÜNDNIS 90/DIE GRÜNEN - Drucksache 19/8145, BTDrs. $19 / 8685$ v. 22.3.2019, S. 7.

20) Zur Staatszielbestimmung Tierschutz: Schürmeier, NuR 2020, $29 \mathrm{ff}$.

21) BT-Drs. $14 / 8860$, S. 3

22) Hirt/Maisack/Moritz, TierSchG, Art. 20a GG, Rdnr. 6.

23) Lorz/Metzger, Tierschutzgesetz, 7. Aufl. 2019, Art. 20a GG, Rdnr. 8 m.w. N.

24) Lorz/Metzger, Tierschutzgesetz, Art. 20a GG, Rdnr. 9; Caspar/ Geissen, NVwZ 2002, 913, 914.

25) Hirt/Maisack/Moritz, TierSchG, Art. 20a GG, Rdnr. 20.

26) Vgl. hierzu eingehend: Bruhn/Wollenteit, NuR 2018, $160 \mathrm{ff}$.

27) Hirt/Maisack/Moritz, TierSchG, Art. 20a GG, Rdnr. 21.

28) Hirt/Maisack/Moritz, TierSchG, Art. 20a GG, Rdnr. 21 mit Verweis auf BT-Drs. 13/7015, S. 2.

29) Hirt/Maisack/Moritz, TierSchG, Art. 20a GG, Rdnr. 8; Jarass/ Pieroth, GG, 16. Aufl. 2020, Art. 20a, Rdnr. 14

30) Hirt/Maisack/Moritz, TierSchG, Art. 20a GG, Rdnr. 8.

31) So schon Wollenteit/Lemke, NuR 2013, 177, 180; Felde, Rechtsgutachten, S. $12 \mathrm{f}$. 
TierSchG vorgesehene verhaltensgerechte Unterbringung. ${ }^{32}$ Maßstab der Beurteilung ist hier das Legehennen-Urteil des BVerfG, das sich bereits im Jahr 1999 mit der artgerechten Haltung von Legehennen befasst und die Hennenhaltungsverordnung ${ }^{33}$, die auf den Schutz von Legehennen bei Käfighaltung abzielt, für nichtig erklärt hat. ${ }^{34}$ Das Urteil des BVerfG ist von allgemeiner Bedeutung und bezieht sich über die Haltung von Legehennen hinaus auf jede Tierhaltung. ${ }^{35}$

Das Gericht stellte in seinem Urteil fest, dass die in der Verordnung getroffenen Regelungen über den Platzbedarf im Käfig und den Zugang zum Futtertrog den Vorgaben in der gesetzlichen Ermächtigung nicht entsprechen. In den Bestimmungen werden nach Ansicht des BVerfG Belange des ethisch begründeten Tierschutzes über die Grenzen eines angemessenen Ausgleichs zurückgedrängt. ${ }^{36}$ So weise die Käfighaltung im Vergleich zu anderen Haltungsformen aus verhaltenswissenschaftlicher Sicht Nachteile auf. Die in einem engen Drahtbehältnis untergebrachten Tiere würden nicht nur an der Ausübung natürlicher Verhaltensformen, wie z. B. Flügelschläge, gehindert. Auch andere arttypische Verhaltensweisen, beispielsweise das Scharren oder die Eiablage an geschützter Stelle in einem Nest, werden gänzlich unterbunden. ${ }^{37}$

Wie die Legehennen in Käfighaltung werden auch die Sauen im Kastenstand nicht artgerecht untergebracht, mit all den bereits dargestellten Folgen für die körperliche und psychische Gesundheit der Tiere. Daher ist diese Haltungsform über einen erheblichen Zeitraum hinweg keine verhaltensgerechte Unterbringung im Sinne von $\$ 2$ Nr. 1 TierSchG. ${ }^{38}$

\subsection{Rechtsprechung zur Beschaffenheit von Kastenständen}

In den vergangenen Jahren war die Kastenstandhaltung Gegenstand (höchst)richterlicher Rechtsprechung. Dabei ging es allerdings nicht um die tierschutzrechtliche Zulässigkeit dieser Haltungsform an sich, sondern lediglich um die Beschaffenheit der Kastenstände und die Frage der Geltung der Regelungen in $\$ 24$ Abs. 4 Nr. 2 TierSchNutztV für jeden Kastenstand und jedes einzelne Schwein, konkret darum, dass ein Schwein im Kastenstand in Seitenlage liegen und seine Beine ausstrecken können muss.

Das VG Magdeburg entschied ${ }^{39}$ im Jahr 2014, dass die anlagenbezogene Regelung des $\$ 24$ Abs. 4 Nr. 2 TierSchNutztV den Tierhalter verpflichte, tierschutzgerechte Kastenstände als Haltungseinrichtungen jederzeit ohne Unterbrechung im Rahmen der ständig aufeinanderfolgenden Zucht- und Haltungszyklen auf Dauer vorzuhalten. ${ }^{40}$ Dem Urteil liegt die Klage eines Schweinehalters zugrunde, der sich gegen einen Bescheid wendete, durch den diverse tierschutzrechtliche Anordnungen verfügt wurden. ${ }^{41}$ Mit dem Bescheid wurde durch den Beklagten unter gleichzeitiger Anordnung der sofortigen Vollziehung angeordnet, ,die Klägerin habe bis Ende 2012 alle belegten Kastenstände so zu gestalten, dass jedes Schwein ungehindert aufstehen, sich hinlegen sowie den Kopf und in Seitenlage die Gliedmaßen ausstrecken könne. " ${ }^{42}$

In seinem Urteil weist das VG Magdeburg insbesondere darauf hin, dass Kastenstände dem $\$ 24$ Abs. 4 Nr. 2 TierSchNutztV dann nicht genügen, ,wenn sie nach Länge oder Breite so ausgelegt sind, dass die Tiere an die Kastenstände anstoßen müssen, bzw. dass ihre Gliedmaßen im Liegen über die Kastenstände hinaus in den Bereich der angrenzenden Kastenstände hineinragen ...".43 Weiter führt das Gericht aus, dass das Stockmaß eines Tieres eine geeignete Grundlage für die Bemessung der notwendigen Breite eines Kastenstandes sei, weil es gewährleiste, dass das Tier in Seitenlänge die Gliedmaßen ausstrecken könne, ohne über Gitter oder Kastenstände hinaus in angrenzende Kastenstände hineinzuragen.
Nachdem die Klage vor dem VG Magdeburg scheiterte, hat die Klägerin Berufung eingelegt, die das OVG Magdeburg mit Urteil ${ }^{44}$ aus dem Jahr 2015 als unbegründet zurückgewiesen hat. Das OVG Magdeburg hat die Entscheidung der Vorinstanz bestätigt und festgestellt, dass nach $\$ 24$ Abs. 4 Nr. 2 TierSchNutztV Kastenstände so beschaffen sein müssen, dass jedes Schwein in Seitenlage die Gliedmaßen ausstrecken kann. ${ }^{45}$ Ebenso führt das Gericht aus, dass die Vorgabe des $\$ 24$ Abs. 4 Nr. 2 TierSchNutztV nur Kastenstände erfüllen, deren Breite mindestens dem Stockmaß des darin untergebrachten Schweins entspricht, oder Kastenstände, welche dem Tier die Möglichkeit eröffnen, die Gliedmaßen ohne Behinderung in die beiden benachbarten leeren Kastenstände oder beidseitig (unbelegten) Lücken durchzustecken. Die Möglichkeit, die Gliedmaßen in benachbarte belegte Kastenstände auszustrecken, sei aber nicht ausreichend. ${ }^{46}$ Die Rechtsprechung des OVG Magdeburg schien für die Schweinehalter weitreichende Konsequenzen zu haben: Entweder die Kastenstände werden so umgebaut, dass sich die jeweilige Sau in dem Kastenstand ausstrecken kann, oder die jeweils benachbarten Kastenstände müssen leer bleiben. ${ }^{47}$

Das BVerwG hat mit seinem Beschluss ${ }^{48}$ im Jahr 2016 entschieden, dass die in $\$ 24$ Abs. 4 Nr. 2 TierSchNutztV enthaltenen Anforderungen an die Beschaffenheit von Kastenständen gegenüber jedem einzelnen in einem Kastenstand gehaltenen Schwein zu erfüllen sind. Die Möglichkeit eines jeden Schweins, sich in einem Kastenstand hinzulegen und in Seitenlage die Gliedmaßen auszustrecken, muss nach Auffassung des BVerwG jederzeit ungehindert gegeben sein und darf nicht auf eine Seite beschränkt werden. Mit seinem Beschluss spricht sich das BVerwG gegen die Durchführung eines Revisionsverfahrens aus und bestätigt die Entscheidung des OVG Magdeburg. ${ }^{49}$

Allerdings stellt das BVerwG auf bauliche Vorgaben der Tierschutz-Nutztierhaltungsverordnung ab und versagt sich den rechtlich gebotenen Rückgriff auf die tierschutzrechtliche Grundnorm des $\$ 2$ TierSchG. ${ }^{50}$ Eine grundsätzliche tierschutzrechtliche Bewertung der Kastenstandhaltung bleibt damit aus.

Wenn die Rechtsprechung auch Vorgaben zur baulichen Beschaffenheit von Kastenständen gemacht hat, zeigte dies

32) Opitz, Tierschutzrechtliche und tierschutzfachliche Aspekte der Kastenstandhaltung von Sauen, in: Rackwitz/Pees (Hrsg.), LBH: 9. Leipziger Tierärztekongress - Tagungsband 3, S. 477, 478.

33) Verordnung des Bundesministeriums für Ernährung, Landwirtschaft und Forsten zum Schutz von Legehennen bei Käfighaltung (Hennenhaltungsverordnung) v. 10.12.1987, BGBl. S. 2622.

34) BVerfG, Urt. v. 6.7.1999, Az: 2 BvF 3/90 (juris).

35) Hirt/Maisack/Moritz, TierSchG, $\$ 2$ TierSchG, Rdnr. 12.

36) BVerfG, Urt. v. 6.7.1999, Az: 2 BvF 3/90, Orientierungssatz 4a (juris).

37) BVerfG Urt. v. 6.7.1999, Az: 2 BvF 3/90, Rdnr. 7 (juris).

38) Eingehend hierzu: Felde, NVwZ 2017, 368, 369.

39) VG Magdeburg, Urt. v. 3.3.2014, Az: 1 A 230/14 (juris).

40) Zur Rechtsprechung zur Ausgestaltung von Kastenständen für Sauen eingehend: Maisack, NuR, 2017, $456 \mathrm{ff}$.

41) VG Magdeburg, Az: 1 A 230/14, Rdnr. 1 (juris).

42) VG Magdeburg, Az: 1 A 230/14, Rdnr. 3 (juris).

43) Hierzu und zum Folgenden VG Magdeburg, Az: 1 A 230/14, Rdnr. 26 (juris).

44) Vom 24.11.2015, Az: 3 L 386/14 (juris).

45) OVG Magdeburg, Urt. v. 24.11.2015 Az: 3 L 386/14, Rdnr. 40 (juris).

46) OVG Magdeburg, Urt. v. 24.11.2015 Az: 3 L 386/14, Rdnr. 41 f. (juris).

47) Dombert/Witt, Münchener Anwaltshandbuch Agrarrecht, 2. Aufl. 2016, \$22 Tierseuchen-, Tierzucht und Tierschutzrecht, Rdnr. 244

48) Vom 8.11.2016, Az. 3 B 11.16, Leitsatz.

49) Eingehend hierzu auch: Maisack, NuR 2017, 456, 459.

50) Felde, NVwZ 2017, 368. 
über Jahre hinweg nur wenige Auswirkungen auf die tatsächlichen Haltungsbedingungen.

\section{Die Siebte Änderungsverordnung}

Als Folge des Beschlusses des BVerwG zur Kastenstandhaltung ${ }^{51}$ aus dem Jahr 2016 wurde dem Bundesrat im November 2019 die Siebte Verordnung zur Änderung der Tierschutz-Nutztierhaltungsverordnung des Bundesministeriums für Ernährung und Landwirtschaft zur Zustimmung vorgelegt. ${ }^{52}$ In seiner Sitzung ${ }^{53}$ im Juli 2020 hat der Bundesrat beschlossen, der Verordnung zuzustimmen. ${ }^{54}$ Das Bundesministerium für Ernährung und Landwirtschaft als Verordnungsgeber erachtet die Anderungsverordnung als Fortschritt, auch wenn dieser erst nach Ablauf der vorgesehenen Übergangsfrist eintrete. ${ }^{55}$ Mit dem Beschluss der Siebten Änderungsverordnung sind Änderungen der auf die Haltungsform des Kastenstands bezogenen Regelungen vorgesehen. Diese betreffen insbesondere die $\$ \$ 24$ und 30 TierSchNutztV sowie die in $\$ 45$ TierSchNutztV vorgesehenen Übergangsregelungen.

\section{1 Änderung des $\int 24$ TierSchNutztV}

Mit der Beschlussfassung der Siebten Änderungsverordnung wird der $\$ 24$ Abs. 4 TierSchNutztV, der bislang die Beschaffenheit der Kastenstände regelte, aufgehoben. ${ }^{56} \$ 24$ Abs. 3 TierSchNutztV wird ein Satz 3 hinzugefügt, wonach der Kastenstand so beschaffen sein muss, dass dem Schwein eine uneingeschränkt nutzbare Bodenfläche zur Verfügung steht, die eine Länge von mindestens 220 Zentimetern aufweist. Zur Breite des Kastenstandes wird in \$24 TierSchNutztV allerdings keine Regelung mehr getroffen. ${ }^{57}$

Das zuständige Bundesministerium weist selbst darauf hin, dass Kastenstände, die der Auslegung des $\$ 24$ Abs. 4 TierSchNutztV a.F. durch das OVG Magdeburg entsprechen, kaum anzutreffen seien. ${ }^{58}$ Daraus lässt sich schlussfolgern, dass ein Großteil der Kastenstände im Bereich des Deckzentrums offensichtlich seit Jahrzehnten nicht anforderungsgerecht gestaltet ist. Damit werden bekannte rechtswidrige Zustände akzeptiert und schließlich auch legalisiert, obwohl das OVG Magdeburg in seiner Entscheidung deutlich gemacht hat, dass die Anforderungen an die Beschaffenheit eines Kastenstandes in $\$ 24$ Abs. 4 Nr. 2 TierSchNutztV zwingend einzuhalten seien.

\section{2 Änderung des $\int 30$ TierSchNutztV}

Die Vorschrift regelt in der bisherigen Fassung im Wesentlichen die Voraussetzungen für eine Gruppenhaltung, und zwar im Zeitraum ab dem ersten Tag der fünften Woche nach dem Belegen bis eine Woche vor dem voraussichtlichen Abferkeltermin. ${ }^{59}$ In Kastenständen dürfen Jungsauen und Sauen nur gehalten werden, wenn nicht offensichtlich erkennbar ist, dass diese Haltungsform zu nachhaltiger Erregung führt, die insbesondere durch Gabe von Beschäftigungsmaterial nicht abgestellt werden kann ( $\$ 30$ Abs. 4 TierSchNutztV).

Durch die Siebte Änderungsverordnung sollen die Absätze $2 \mathrm{a}$ und $2 \mathrm{~b}$ neu in $\$ 30$ TierSchNutztV aufgenommen werden. Während in Abs. 2a die bauliche Gestaltung der Gruppenhaltung geregelt wird, nimmt Abs. 2b Bezug auf die Kastenstandhaltung. Dieser sieht in Satz 2 vor, dass Jungsauen und Sauen nur für einen Zeitraum von längstens fünf Tagen, der die Zeit beinhaltet, in der die Jungsau oder Sau abferkelt, im Kastenstand gehalten werden dürfen.

\section{3 Übergangsregelungen}

Auch $\$ 45$ TierSchNutztV wird mit der Beschlussfassung der Verordnung geändert. ${ }^{60}$ Der neue Absatz 11a erlaubt übergangsweise unter bestimmten Bedingungen die Haltung von Jungsauen und Sauen in Haltungseinrichtungen, die vor dem 9. Februar 2021 bereits genehmigt oder in Benutzung genommen worden sind, noch bis zum Beginn des 9. Februar 2029. Voraussetzung ist, dass die Tiere im Zeitraum von über vier Wochen nach dem Decken bis eine Woche vor dem voraussichtlichen Abferkeltermin in der Gruppe gehalten werden. Zudem müssen die Kastenstände im Übergangszeitraum so beschaffen sein, dass die Schweine sich nicht verletzen können, jedes Schwein ungehindert aufstehen, sich in Seitenlage hinlegen sowie den Kopf ausstrecken kann und jedes Schwein seine Gliedmaßen in Seitenlage ausstrecken kann, ohne dass dem ein bauliches Hindernis entgegensteht. ${ }^{61}$ Der jeweilige Tierhalter ist des Weiteren verpflichtet, der zuständigen Behörde bis zum 9. Februar 2024 ein Betriebs- und Umbaukonzept der vorhandenen Haltungseinrichtungen sowie bis zum 9. Februar 2026 den Nachweis über einen ggf. gestellten Bauantrag vorzulegen. Diese Übergangsregelung gilt für das Deckzentrum. Auf Antrag des Tierhalters kann die Weiternutzung einer bestehenden Haltungseinrichtung zur Vermeidung einer unbilligen Härte auf zehn Jahre ausgedehnt werden. Sofern der Tierhalter bis zum 9. Februar 2024 gegenüber der zuständigen Behörde verbindlich erklärt, dass er die Haltung von Schweinen spätestens zum 9. Februar 2026 endgültig einstellen wird, entfällt die Pflicht zur Vorlage eines Umbaukonzepts ( $\$ 45$ Abs. 11a Satz 3 TierSchNutztV).

Der neu eingefügte $\int 45$ Abs. 11b TierSchNutztV ${ }^{62}$ bezieht sich auf die Kastenstände, die sich in Abferkelbuchten befinden. Auch dieser Absatz enthält eine Übergangsregelung, die allerdings eine Übergangszeit von 15 Jahren für bestehende Haltungseinrichtungen vorsieht, also bis zum Beginn des 9. Februar 2036. Dies gilt unter der Voraussetzung, dass die Jungsauen und Sauen im Zeitraum von über vier Wochen nach dem Decken bis eine Woche vor dem Abferkeltermin in der Gruppe gehalten werden, die Kastenstände der Abferkelbuchten so beschaffen sind, dass die Schweine sich nicht verletzen können und jedes Schwein ungehindert aufstehen, sich hinlegen sowie den Kopf und in Seitenlage die Gliedmaßen ausstrecken kann. Des Weiteren müssen die Abferkelbuchten so angelegt sein, dass hinter dem Liegebereich der Jungsau oder der Sau genügend Bewegungsfreiheit für das ungehinderte Abferkeln sowie für geburtshilfliche Maßnahmen besteht. Der Tierhalter muss der zuständigen Behörde bis zum 9. Februar 2033 ein Betriebs- und Umbaukonzept sowie den Nachweis über einen ggf. erforderlichen Bauantrag vorlegen. Auch der Abs. 11b enthält eine Härtefallregelung, die im Einzelfall eine Übergangsfrist bis zum Beginn des 9. Februar 2038 erlaubt.

51) BVerwG, Beschl. v. 8.11.2016, Az: 3 B 11.16.

52) BR-Drs. 587/19 v. 7.11.2019.

53) 992. Sitzung am 3.7.2020

54) Beschl. des Bundesrates, Siebte Verordnung zur Änderung der Tierschutz-Nutztierhaltungsverordnung, BR-Drs. 302/20, Beschl. v. 3. 7.2020.

55) BR-Drs. 587/19, S. 2

56) In der Grunddrucksache $587 / 19$ war eine Streichung des $\$ 24$ Abs. 4 TierSchNutztV a.F. noch nicht vorgesehen. Vielmehr sollte Abs. 4 geändert werden.

57) Hingegen enthielt der Verordnungsentwurf v. 7.11.2019 (BRDrs. 587/19) in $\$ 24$ Abs. 4 konkrete Angaben zur Breite eines Kastenstandes im Deckzentrum

58) BR-Drs. 587/19, S. 11 (Begründung).

59) Hirt/Maisack/Moritz, TierSchG, TierSchNutztV, $₫ 30$, Rdnr. 1.

60) BR-Drs. 302/20 (Beschluss), S. 7.

61) Beschluss der Siebten Verordnung zur Änderung der TierschutzNutztierhaltungsverordnung v. 3.7.2020, BR-Drs. 302/20 (Beschluss), S. $7 \mathrm{f}$.

62) BR-Drs. 302/20 (Beschluss), S. 11. 


\subsection{Rechtliche Bewertung der Änderungen}

Zwar sieht die Siebte Änderungsverordnung eine Abschaffung der Kastenstandhaltung im Deckzentrum vor, allerdings mit der Einschränkung, dass eine Fixation der Sauen im Rahmen des Reproduktionszyklus zum Zeitpunkt der Besamung noch zulässig ist. ${ }^{63}$

Daneben soll die Dauer der Fixierung der Sauen im Abferkelbereich verkürzt werden. Dennoch bringen die langen Übergangszeiten bis zur Umstellung der Haltung für das einzelne Tier über Jahre hinweg keine Änderung.

Das Bundesministerium für Ernährung und Landwirtschaft hat ein Förderprogramm zum Stallumbau ${ }^{64}$ mit dem Ziel aufgelegt, im Interesse des Tierwohls die Haltungsbedingungen möglichst vor Ablauf der festgelegten Übergangsfristen umzusetzen. Dennoch wird es Jahre dauern, bis die Kastenstandhaltung flächendeckend abgeschafft sein wird.

Im Hinblick auf die Regelung zur Beschaffenheit des Kastenstandes im Deckzentrum wird eine bis dato bestehende Regelung zum ungehinderten Ausstrecken der Beine des Schweines aus der Tierschutz-Nutztierverordnung gestrichen ( $\$ 24$ Abs. 4 Nr. 2 TierSchNutztV a. F.). Für die Ubergangszeit gilt eine Regelung, die ein Ausstrecken der Gliedmaßen der Sauen nicht uneingeschränkt ermöglicht, zuma lediglich kein bauliches Hindernis dem Ausstrecken entgegenstehen darf. Sind die benachbarten Kastenstände belegt, ist das Ausstrecken der Gliedmaßen nicht ungehindert möglich. In dieser Übergangsregelung zu Lasten der Tiere liegt ein Verstoß gegen das in Art. 20a GG enthaltene Verschlechterungsverbot. Um der Staatszielbestimmung Tierschutz gerecht $\mathrm{zu}$ werden, hätten die Regelungen zur Beschaffenheit des Kastenstandes im Deckzentrum ( $\$ 24$ Abs. 4 Nr. 2 TierSchNutztV a.F.) zumindest beibehalten und in der Praxis konsequent nach der Rechtsprechung des OVG Magdeburg durchgesetzt werden müssen. Eine wirkliche Verbesserung für die Millionen Zuchtsauen wäre nach Jahrzehnten der Kastenstandhaltung erreicht worden, wenn diese Haltungsform innerhalb einer deutlich kürzeren Übergangsfrist abgeschafft worden wäre. In der Vergangenheit war lange Zeit, um sich auf einen solchen Ausstieg vorbereiten zu können.

\section{Der Normenkontrollantrag des Landes Berlin}

Die Berliner Landesregierung hat am 14. Januar 2019 einen Normenkontrollantrag beim Bundesverfassungsgericht eingereicht, der sich gegen Regelungen der TierschutzNutztierhaltungsverordnung wendet. Als sog. Blaupause dient dem Land Berlin das Normenkontrollverfahren aus Nordrhein-Westfalen in den 90er Jahren zur Legehennenhaltung, das zu einer Veränderung geführt habe. ${ }^{65}$

Das Land Berlin begründet den Antrag damit, dass es zentrale rechtliche Anforderungen an die Schweinehaltung in der deutschen Landwirtschaft für verfassungswidrig hält. ${ }^{66}$ Die Tierschutz-Nutztierhaltungsverordnung lasse es nach Ansicht des Landes zu, ,dass Grundbedürfnisse der Tiere in einer Weise eingeschränkt werden, die mit den Vorgaben des Tierschutzgesetzes (TierSchG) nicht vereinbar ist" ${ }^{67}$ Als ein Aspekt der Schweinehaltung wird die Zulässigkeit der mehrwöchigen Fixierung von Sauen in Kastenständen im Deck- und Abferkelbereich thematisiert, wodurch insbesondere das Bedürfnis nach Sozialkontakt, nach Mutter-Kind-Verhalten und Schlafen/Ruhen weitgehend unterdrückt wird ( $₫ 30$ Abs. 2 Satz 1 und Abs. 4 TierSchNutztV). ${ }^{68}$ Das Land Berlin ist der Auffassung, dass diese Regelung mit der gesetzlichen Ermächtigungsgrundlage des $\$ 2$ a TierSchG i. V.m. $\$ 2$ TierSchG als höherrangigem Recht unvereinbar und deshalb nichtig ist. ${ }^{69}$

\section{Zusammenfassung und Ausblick}

Die Siebte Änderungsverordnung der Tierschutz-Nutztierhaltungsverordnung zielt auf eine Abschaffung der Kastenhaltung im Deckzentrum und eine deutliche Reduzierung der Fixierungszeit in einem Kastenstand im Abferkelbereich $\mathrm{ab}$. In der Sache ist die Initiative des Verordnungsgebers zu begrüßen, wird allerdings durch die langen Übergangsfristen konterkariert. Die erhoffte und aus tierschutzrechtlicher Sicht zeitnahe Abschaffung der Kastenstandhaltung bleibt also aus, mit der Folge, dass noch Generationen von Sauen durch die Kastenstandhaltung leiden.

Wenn der Verordnungsgeber die Kastenstandhaltung schon nicht sofort abschaffen will, so hätte er zumindest die höchstrichterliche Entscheidung zur Beschaffenheit der Kastenstände umsetzen können. Stattdessen wurde die Regelung den Zuständen in den in den Ställen angepasst. Vor diesem Hintergrund ist es zu begrüßen, dass der Normenkontrollantrag des Landes Berlin auch die Kastenstandhaltung in Deckzentrum und Abferkelbereich einbezieht.

Auch für andere Formen der Massentierhaltung besteht aus regulatorischer Sicht wenig Hoffnung, es sei denn, das Verbraucherverhalten honoriert tierwohlverträgliche Haltungsbedingungen auch mit Blick auf die Marktpreise.

Open Access. Dieser Artikel wird unter der Creative Commons Namensnennung 4.0 International Lizenz veröffentlicht, welche die Nutzung, Vervielfältigung, Bearbeitung, Verbreitung und Wiedergabe in jeglichem Medium und Format erlaubt, sofern Sie den/die ursprünglichen Autor(en) und die Quelle ordnungsgemäß nennen, einen Link zur Creative Commons Lizenz beifügen und angeben, ob Änderungen vorgenommen wurden.

Die in diesem Artikel enthaltenen Bilder und sonstiges Drittmaterial unterliegen ebenfalls der genannten Creative Commons Lizenz, sofern sich aus der Abbildungslegende nichts anderes ergibt. Sofern das betreffende Material nicht unter der genannten Creative Commons Lizenz steht und die betreffende Handlung nicht nach gesetzlichen Vorschriften erlaubt ist, ist für die oben aufgeführten Weiterverwendungen des Materials die Einwilligung des jeweiligen Rechteinhabers einzuholen.

Weitere Details zur Lizenz entnehmen Sie bitte der Lizenzinformation auf http://creativecommons.org/licenses/by/4.0/deed.de.

Open Access funding enabled and organized by Projekt DEAL.

63) BR-Drs. 302/20 (Beschluss), S. 14.

64) Bundesministerium für Ernährung und Landwirtschaft, Richtlinie zur Förderung des Stallumbaus zur Verbesserung der Haltungsbedingungen von Sauen v. 8.11.2020, BAnz AT 16.9.2020 B2.

65) DJGT, Bericht über die Pressekonferenz zum Normenkontrollantrag des Landes Berlin beim Bundesverfassungsgericht zur Schweinehaltung am 9.1.2019, Stand 20.6.2021, abrufbar unter https://www.vereinonline.org/djgt/files/www/veroeffentlichungen33.pdf, S. 2,

66) Vgl. Zusammenfassung des Normenkontrollantrags der Berliner Senatsverwaltung für Justiz, Verbraucherschutz und Antidiskriminierung, S. 1, E-Mail der Senatsverwaltung für Justiz, Verbraucherschutz und Antidiskriminierung (Pressestelle) v. 7.1.2021. Vgl. auch Senatsverwaltung für Justiz, Verbraucherschutz und Antidiskriminierung, Berlin hält an Normenkontrolle zur Schweinehaltung fest, Pressemitteilung v. 3.7.2020, Stand 20.6.2021, abrufbar unter https://www.berlin.de/sen/justva/ presse/pressemitteilungen/2020/pressemitteilung.955782.php.

67) Zusammenfassung, S. 1

68) Zusammenfassung, S. 1.

69) Zusammenfassung, S. 2. 